Antibody response and reactions to aqueous influenza vaccine, simple emulsion vaccinc
and multiple emulsion vaccine. A report to the Medical
Research Council Committee on influenza and
other respiratory virus vaccines*

\author{
BY P. J. TAYLOR $\dagger$ \\ T.U.C. Centenary Institute of Occupational Health \\ CHRISTINE L. MILLER AND T. M. POLLOCK \\ Epidemiological Research Laboratory, Public Health Laboratory Service \\ AND F. T. PERKINS AND M. A. WESTWOOD
}

Medical Research Council, London

(Received 15 January 1969)

Influenza vaccines prepared with a mineral oil adjuvant induce a substantial and durable antibody response. However, vaccines containing mineral oil in simple emulsion (water-in-oil emulsion) sometimes produce persistent local reactions (Medical Research Council, 1964). The development of a redispersed 'multiple' emulsion in which antigen is incorporated as an oil-in-water emulsion starting from an original water-in-oil emulsion was described by Herbert (1965). Such multiple emulsion vaccine has a lesser viscosity than simple emulsions and might therefore produce a substantial antibody response with fewer reactions. In this investigation a comparison has been made of the antibody response and short-term vaccination reactions after aqueous influenza vaccine, influenza vaccine containing a mineral oil in simple emulsion, and influenza vaccine containing mineral oil in multiple emulsion.

\title{
GENERAL PLAN
}

The investigation began in November 1966. There were approximately 300 participants all of whom were volunteers employed at the oil refinery at Shell Haven, Essex. They were aged 20-59 years. A blood sample was withdrawn from each volunteer and, immediately after, each received an intramuscular injection of one of the following four vaccines: either aqueous vaccine, or adjuvant simple emulsion vaccine, or adjuvant multiple emulsion vaccine, or, as a control, rhinovirus

* Members of Committee: Prof. C. H. Stuart-Harris (Chairman), Dr A. S. Beare, Prof. G. Belyavin, Dr J. T. Boyd, Prof. G. W. A. Dick, Prof. Sir Austin Bradford Hill, Dr F. Himmelweit, Dr D. Hobson, Dr W. W. Holland, Sir James Howie, Dr F. O. MacCallum, Dr H. G. Pereira, Dr F. T. Perkins, Dr T. M. Pollock, Dr A. T. Roden, Dr D. A. J. Tyrrell.

Requests for reprints should be addressed to: Dr P. J. Taylor, London School of Hygiene and Tropical Medicine, Keppel Street, London, W.C. 1.

$\dagger$ Previously Shell U.K. Ltd, Shell Haven. 
vaccine. The three influenza vaccines each contained identical virus strains from the same virus pool. The four vaccines were given in strict rotation in the order in which each volunteer arrived at the clinic.

Twenty-four hours after vaccination as many as possible of the participants were seen and asked about the occurrence of systemic reactions and the vaccination sites were examined. Thereafter, each participant was invited to complete and return a questionnaire stating whether or not any reactions occurred $72 \mathrm{hr}$. after vaccination and giving appropriate descriptions; those who failed to return the questionnaire were followed up and examined. Further blood samples were withdrawn approximately 3 and 12 months after vaccination. The haemagglutination-inhibiting (HI) antibody titres in the pre-vaccination serum and the two postvaccination sera were titrated in parallel. During the 3 months after vaccination an account was kept of the sickness absence of the volunteers.

\section{The vaccines}

\section{METHODS}

These were prepared by Evans Medical Ltd.

Aqueous vaccine. The aqueous vaccine was a saline suspension of the following composition (given in haemagglutinating units (Salk)): $\mathrm{A}_{2} /$ England/12/64, 5250; $\mathrm{A}_{2} /$ England/76/66, 2500; B/England/939/59, 3250; B/England/5/66, 3250. The material was inactivated by the use of formalin (1/4000) followed by betapropiolactone (1/1000), concentrated by differential centrifugation and, after appropriate dilution in phosphate buffered saline containing $0.013 \%$ thiomersal, was distributed into $1 \mathrm{ml}$. ampoules.

Simple emulsion. Using the same strains, a 50/50 water-in-oil emulsion was prepared with Drakeol 6 VR and Arlacel A. (The constituents of the emulsion and the final vaccine passed the tests described by Berlin (1962).) The emulsion was then dispensed into disposable syringes so that a dose of $0.25 \mathrm{ml}$. contained one quarter of the haemagglutinin content of the simple aqueous vaccine for each strain.

Multiple emulsion. This was prepared by the addition of one-half volume of phosphate saline containing Tween 80 to 1 volume of simple water in oil/oil in water emulsion. The mixture was then re-emulsified to produce a double emulsion. After emulsification the material was dispensed into disposable syringes in $0 \cdot 375 \mathrm{ml}$. amounts to give the same HA content as that of the simple emulsion.

All vaccines were tested for conformity with the Therapeutic Substances Act requirements.

\section{Antibody titrations}

The serological tests were carried out in perspex trays by the method recommended by the World Health Organization Expert Committee on Influenza (1953) using a final dilution of four agglutinating doses of antigen. Readings were made after $60 \mathrm{~min}$. at room temperature, the end-point being taken as $50 \%$ inhibition of agglutination or calculated by interpolation when the $50 \%$ end-point fell between two dilutions. 
All sera were treated with Vibrio cholerae enzyme (R.D.E.) and pre- and postvaccination sera of each individual were always included in the same test.

\section{RESULTS}

\section{Vaccination reactions and sickness absence}

A total of 302 volunteers were vaccinated. Their ages ranged from 20 to 59 years and all but 18 were males.

Of these participants only 162 had all the necessary serum samples withdrawn. Of these so tested 41 received aqueous vaccine; 39 simple emulsion vaccine; 42 multiple emulsion vaccine; and 40 rhinovirus (control) vaccine.

\section{Table 1. Local and general reactions up to $72 \mathrm{hr}$ after vaccination, according to group}

\begin{tabular}{llccc} 
Type of & \multicolumn{4}{c}{ Nos. showing reactions after } \\
\cline { 2 - 5 } reaction & Aqueous & $\begin{array}{c}\text { Simple } \\
\text { emulsion }\end{array}$ & $\begin{array}{c}\text { Multiple } \\
\text { emulsion }\end{array}$ & $\begin{array}{c}\text { Rhinovirus } \\
\text { (control) }\end{array}$ \\
Local & $6(8)$ & $6(8)$ & $20(26)$ & 0 \\
General & $21(27)$ & $27(36)$ & $29(38)$ & $10(14)$ \\
No reaction & $51(65)$ & $41(55)$ & $27(36)$ & $64(86)$ \\
Total & 78 & 74 & 76 & 74
\end{tabular}

Figures in parentheses indicate percentages.

There were no severe local reactions, but mild erythema, swelling and discomfort were common; none of the reactions was troublesome. The frequency of local reactions after multiple emulsion vaccine was much greater than with the other vaccines, but this increase was noted $24 \mathrm{hr}$. after vaccination only and was a reflexion of the large number of complaints of only slight discomfort from the recipients of this vaccine. Mild general reactions consisting of headache, muscular pain and malaise were common complaints after all the influenza vaccines and were less frequent after rhinovirus vaccine (Table 1).

Ninety-one participants had been immunized about 2 years previously with an influenza vaccine containing an oil adjuvant; the reactions in these participants were not more frequent or severe than those among persons who had not been immunized previously.

During the 4 months after immunization there was no outbreak of influenza and, as might be expected, the time lost by sickness absence from all causes was the same in each of the vaccination groups. This similarity was maintained for the 12 months after inoculation. However, irrespective of the vaccine given the sickness time lost by absence from all causes was greater among participants with reactions after vaccination than among those with no reactions. Thus of the 119 participants with a reaction, either local or general, $48(40 \%)$ had at least one spell of sickness during the 3 months after vaccination, whereas of the 183 persons without reactions, $48(26 \%)$ had at least one spell of sickness. These differences attain statistical significance at the $5 \%$ level. 


\section{Antibody titres before vaccination}

Each of the vaccine groups contained participants with and without detectable antibody (Table 2).

Fifty-three participants from whom serum samples were obtained had been vaccinated 2 years previously with oil adjuvant vaccine containing $3500 \mathrm{HI}$ units of the following four virus strains: A/Singapore/1/57, B/England/939/59, A/England/ $1 / 61$ and $B /$ Taiwan $/ 4 / 62$.

Of the 53 participants who had been previously vaccinated nearly $91 \%$ had antibody to the A 2 virus and $66 \%$ antibody to the B virus (Table 3) whereas only about $40 \%$ of persons who were not vaccinated had antibody to $\mathrm{A} 2$ virus and $23 \%$ antibody to the $B$ virus.

Table 2. Antibody titres before vaccination according to group

\begin{tabular}{|c|c|c|c|c|c|c|c|c|c|}
\hline \multirow[b]{3}{*}{ Group } & \multirow[b]{3}{*}{ No. } & \multicolumn{8}{|c|}{ Antibody titres } \\
\hline & & \multicolumn{2}{|c|}{$<12$} & \multicolumn{2}{|c|}{$12-48$} & \multicolumn{2}{|c|}{$>48$} & \multicolumn{2}{|c|}{$\begin{array}{l}\text { Geometric } \\
\text { mean titres }\end{array}$} \\
\hline & & A 2 & B & A 2 & B & A 2 & B & A 2 & B \\
\hline Aqueous & 41 & 20 & 27 & 15 & 11 & 6 & $\mathbf{3}$ & 16 & 10 \\
\hline Simple emulsion & 39 & 17 & 24 & 12 & 12 & 10 & 3 & 20 & 12 \\
\hline Multiple emulsion & 42 & 18 & 27 & 19 & 13 & 5 & 2 & 17 & 10 \\
\hline Rhinovirus (control) & 40 & 16 & 24 & 12 & 12 & 12 & 4 & 25 & 13 \\
\hline
\end{tabular}

Table 3. Antibody titres in first serum sample according to history of previous influenza vaccination

\begin{tabular}{|c|c|c|c|c|c|c|c|c|}
\hline \multirow[b]{3}{*}{ Group } & & & \multicolumn{6}{|c|}{ Antibody titres } \\
\hline & \multicolumn{2}{|c|}{ Total no. } & \multicolumn{2}{|c|}{$<12$} & \multicolumn{2}{|c|}{$12-48$} & \multicolumn{2}{|c|}{$>48$} \\
\hline & A2 & B & A2 & B & A 2 & B & A2 & B \\
\hline $\begin{array}{l}\text { Previously } \\
\text { vaccinated }\end{array}$ & 53 & 53 & $5(9)$ & $18(34)$ & $22(42)$ & $24(45)$ & $26(49)$ & $11(21)$ \\
\hline $\begin{array}{l}\text { Not previously } \\
\text { vaccinated }\end{array}$ & 109 & 109 & $66(61)$ & $84(77)$ & $36(33)$ & $24(22)$ & $7(6)$ & $1(1)$ \\
\hline
\end{tabular}

Figures in parentheses indicate percentages.

\section{Antibody response to vaccination}

The geometric means of the antibody titres before vaccination and at approximately 3 and 12 months after vaccination are shown in Table 4 . It is evident that there was a very substantial increase in antibody to the A 2 component after each of the three influenza vaccines, but that this increase was not the same after each vaccine. Three months after aqueous vaccine the mean titre was approximately 11 times greater than the prevaccination sample; after simple emulsion vaccine 16 times greater; and after multiple emulsion vaccine 27 times greater. One year after vaccination the titres produced by each vaccine had declined substantially to roughly half the titres observed at 3 months. However, of those without antibody 
before vaccination and who thus might be expected to be specially susceptible to a natural infection with influenza virus, 18 of 20 given aqueous vaccine possessed antibody to the A 2 virus at the end of 1 year. All 17 participants without initial antibody who were given simple emulsion vaccine and all 18 participants without initial antibody given multiple emulsion vaccine had antibody at 1 year.

Table 4. Geometric mean of antibody titres in 1 st, 2 nd and 3 rd serum samples, i.e. immediately before vaccination, approximately three months after vaccination and approximately 12 months after vaccination

\begin{tabular}{llrrrrrrr}
\multicolumn{1}{c}{ Vaccine } & No. & $\overbrace{\text { 1st }}$ & 2nd & 3rd & $\overbrace{\text { 1st }}^{\text {A2 }}$ & 2nd & 3rd \\
Aqueous & 41 & 16 & 171 & 102 & 10 & 34 & 23 \\
Simple emulsion & 39 & 20 & 334 & 166 & 12 & 89 & 52 \\
Multiple emulsion & 42 & 17 & 461 & 206 & 10 & 132 & 70 \\
Rhinovirus (control) & 40 & 25 & 26 & 25 & 13 & 13 & 12
\end{tabular}

The mean antibody titre to the influenza $B$ virus component showed a much smaller increase at 3 months than the increase to the $A 2$ virus component, but the increase was less after aqueous vaccine (approximately three-fold) than to the simple emulsion and multiple emulsion vaccines (seven-fold and thirteen-fold respectively). With each vaccine the mean titre declined 1 year after vaccination. For those without initial influenza $B$ antibody titres 15 of 27 given aqueous vaccine, 22 of 24 given simple emulsion vaccine and 25 of 27 given multiple emulsion vaccine had antibody at 12 months. The antibody response in the recipients of the influenza vaccine was not due to natural infection since the geometric mean titres of the control group showed little alteration throughout.

\section{COMMENT}

In this investigation the influenza vaccine under test contained a mineral oil adjuvant, Drakeol $6 \mathrm{VR}$ and Arlacel A, prepared as a multiple emulsion. This preparation is less viscid than a simple emulsion preparation and might thus be less likely to produce local reactions; on the other hand it might also produce a less intense or durable antigenic stimulus. The multiple emulsion preparation was therefore compared with a vaccine containing the same adjuvants prepared in simple emulsion, and with an aqueous vaccine.

Although reactions to the multiple emulsion vaccine were more frequent than to the aqueous and simple emulsion preparations, the reactions were slight and in themselves would not constitute a serious drawback to the more extensive use of the vaccine. The observation that sickness absence during the subsequent followup was greater among participants who complained about reactions than among those who did not, may reflect the fact that some individuals are constitutionally more inclined to complain of minor symptoms than others, whether these symptoms are the result of vaccination or of natural ills. 
The antibody response induced by the multiple emulsion preparation was very satisfactory, greater than the response to the simple emulsion vaccine and substantially greater than to the aqueous vaccine. The reversion of the emulsion to an oil-in-water suspension did not, therefore, impair its adjuvant properties.

With each vaccine the antibody titres observed soon after vaccination declined substantially by 12 months. Despite this decline and irrespective of the vaccine given, almost all the participants who had no antibody to the A 2 virus before vaccination had antibody at 12 months. Almost all the participants without initial antibody to the influenza $\mathrm{B}$ virus and who received either simple or multiple emulsion vaccine had antibody at 12 months, but this was not so for the corresponding participants given aqueous vaccine.

The findings suggest that the multiple emulsion vaccine is at least as effective as the standard oil-emulsion preparation, but provide no information about the frequency and character of late reactions or of the value of the vaccine for routine use. A prolonged and comprehensive examination of this aspect would be required before its routine use could be considered.

\section{REFERENCES}

Berlin, B. S. (1962). Tests for biologic safety of arlacel A. Ann. Allergy 20, 472.

Herbert, W. J. (1965). Multiple emulsions: a new form of mineral-oil antigen adjuvant. Lancet ii. 771.

Medical Research Council (1964). Clinical trials of oil-adjuvant influenza vaccines. 1960-3. Br. med. J. ii, 267.

World Health Organization (1953). Expert Committee on influenza: First report. Tech. Rep. Ser. Wld Hlth Org. no. 64. 